\section{Safety and efficacy of a $10 \%$ intravenous immunoglobulin preparation in patients with immune thrombocytopenic purpura: results of two international, multicenter studies}

\begin{abstract}
Aim: To assess safety and efficacy of a $10 \%$ intravenous immunoglobulin in patients with primary immune thrombocytopenic purpura (ITP). Patients \& methods: ITP patients in two multicenter studies (Trials A/B) were treated with $2 \mathrm{~g} / \mathrm{kg}$ Flebogamma ${ }^{\circledR}$ 10\% DIF (over 2-5 days) and were followed up to 1-3 months. Results: 18 patients in Trial A and 58 in Trial B were enrolled (12 children in Trial B). The response rate (platelet count $\geq 50 \times 10^{9} / \mathrm{l}$ ) was $72.2 \%$ (Trial A) and 76.1/100\% (adults/children; Trial B). Most patients improved bleedings ( $83.3 \%$ Trial A; $88.9 \%$ Trial B). Potential treatment-related adverse events were reported by $38.9 \%$ (Trial A) and 30.4/83.3\% (adults/children; Trial B) of patients. All serious adverse events (five patients) resolved without sequelae. Conclusion: Flebogamma 10\% DIF was effective and safe in patients with primary ITP.
\end{abstract}

First draft submitted: 7 July 2016; Accepted for publication: 28 September 2016; Published online: 7 November 2016

Keywords: Flebogamma ${ }^{\circledR} 10 \%$ DIF $\bullet$ hemorrhage $\bullet$ immune thrombocytopenic purpura - intravenous immunoglobulin

Immune thrombocytopenic purpura (ITP) is an autoimmune disorder characterized by platelet destruction and thrombocytopenia [1] due to autoantibody binding to platelet antigen(s) causing their premature destruction by reticuloendothelial cells, in particular those of the spleen [2-4]. Previous evidence suggests that platelet production is also decreased in many patients with ITP $[2,5]$.

The diagnosis of ITP remains one of exclusion [2]. Clinical features of ITP are highly variable in ITP subjects ranging from the fairly common asymptomatic presentation with mild bruising and mucosal bleeding to frank hemorrhage from any site. Overall, bleeding signs are uncommon unless ITP is severe (platelet count $<30 \times 10^{9} / \mathrm{l}$ ). The estimated incidence of ITP is one new patient per 100,000 persons per year [4].

The initial approach to the treatment is the use of plasma exchange with fresh frozen plasma or glucocorticoids such as prednisone (1-1.5 mg/kg per day) [2,6]. Response rates range from $50 \%$ to over $75 \%$, depending on the intensity and duration of the therapy, although their effects are inconsistent and often transient. Splenectomy may be considered depending on the severity of the disease, tolerance of corticosteroids and the patient's preferences with regard to surgery. Splenectomy is effective in 50-90\% of patients, and frequently patients present a maintained response with additional treatment [6,7]. Despite conventional therapy, platelet count in ITP patients often remains low, and patient may present bleeding, particularly when platelet count is below $10 \times 10^{9} / 1[6,8]$.

Intravenous immune globulin (IVIG) therapy has been previously shown to be efficacious at increasing platelet counts to hemostatic levels. IVIG treatment at $2 \mathrm{~g} / \mathrm{kg}$ infused over 2-5 days is indicated in children and adults with ITP with high risk of bleeding or prior to surgery to correct platelet count $[2,6,9]$. IVIG treatment can be approached in cases of refractoriness to
Lidia Kovaleva', Shashikant Apte $^{2}$, Sharat Damodar ${ }^{3}$, Vijay Ramanan ${ }^{4}$, Svetlana Loriya $^{\dagger, 5}$, Jordi NavarroPuerto $^{6}$, Ali Khojasteh ${ }^{*}, 7$ $\&$ the investigators of the Flebogamma DIF in ITP Study Group

'Hematology Research Centre of Russian Academy of Medical Science, Moscow, Russia

${ }^{2}$ Sahyadri Speciality Hospital, Pune, Maharashtra, India

${ }^{3}$ Narayana Hrudayalaya Hospitals, Bangalore, Karnataka, India ${ }^{4}$ Ruby Hall Clinic, Pune, Maharashtra, India

${ }^{5}$ Federal Research Clinical Centre of Pediatric Hematology, Oncology \& Immunology, Moscow, Russia ${ }^{6}$ Clinical Trials Department, Instituto Grifols S.A., Barcelona, Spain

${ }^{7}$ Columbia Comprehensive Cancer Care Clinic \& Research Institute, 1705 Christy Dr. Suite 214, Jefferson City, MO 65101, USA

*Author for correspondence:

Tel.: +1 5738936404

Fax: +1 5738936505

drk5c@socket.net

${ }^{+}$Deceased author 
plasma exchange [10]. Among patients with chronic ITP, the increased platelet count is seen in about $75 \%$ of patients and about half of patients reach normal levels [2,5]. Anti-Rh (D) immunoglobulins have also been considered as short-term treatment in $\mathrm{Rh}(\mathrm{D})$-positive ITP patients. The most accepted mechanism of action of IVIG in ITP is the blockade of Fcy-receptors on macrophages, preventing the destruction of IgG-sensitized platelets by the reticuloendothelial system [11]. Several molecular strategies to develop improved IVIG are currently ongoing [12] as well as the use of subcutaneous Ig to autoimmune disease is being explored [13].

Flebogamma ${ }^{\circledR} 10 \%$ DIF is an IVIG $10 \%$ manufactured by Instituto Grifols S.A. (Barcelona, Spain) under the same production process than the $5 \%$ formulation of Flebogamma DIF, which has been described safe and effective for the treatment of ITP [14]. Only during the final concentration step, the final adjustment of the product is performed to generate 5 and $10 \%$ of protein concentrations. Flebogamma $10 \%$ DIF has been licensed in the USA since July 2010 and in Europe since December 2010.

Here, we report the results of two multicenter, prospective, open-label clinical trials performed in six countries worldwide designed to evaluate the safety and the efficacy of Flebogamma 10\% DIF in subjects suffering from primary ITP.

\section{Methods}

\section{Study design}

The overall analysis included two similarly designed open-label, noncontrolled, multicenter clinical trials identified in this paper as Trial A (study ID IG-202; ClinicalTrials.gov Identifier NCT00699140) and Trial B (study ID IG-0601; ClinicalTrials.gov Identifier NCT00511147). Trial A involved seven study sites in Europe (four in Spain, two in Russia, one in the UK) and the Trial B involved 25 study sites in America and Asia (one in Canada, 16 in the USA, eight in India).

In the overall design, primary ITP subjects with severe thrombocytopenia (platelet count $\leq 20 \times 10^{9} / \mathrm{l}$ ) were enrolled (screening visit). After enrollment, IVIG (Flebogamma 10\% DIF; Instituto Grifols S.A.) was infused for a total dose of $2 \mathrm{~g} / \mathrm{kg}$ (over 2 or 5 days). Clinical follow-up of the subjects (up to 1 or 3 months) included efficacy assessments (platelet counts monitoring and regression of bleeding episodes) and safety assessments (adverse events [AEs], physical exams, vital signs and laboratory parameters).

\section{Patients}

Both males and females diagnosed with primary (absence of any obvious initiating and/or underlying cause) ITP and platelet count $\leq 20 \times 10^{9} / 1$ were included. The age range was $18-82$ years in Trial A and 3-70 years in Trial B.

To be eligible, patients at the screening visit had to report an irrelevant medical history and physical examination except for bleeding signs associated with platelet disorders (petechia, purpura, conjunctival hemorrhage or other types of mucocutaneous bleedings); present a normal blood count apart from isolated thrombocytopenia; a peripheral blood smear consistent with ITP (only in Trial A) and a previously known diagnosis of ITP for at least 3 months (Trial A) or 6 months (Trial B) in which at least one episode of thrombocytopenia (platelet count lower than $150,000 / \mu \mathrm{l}$ ) had occurred. Furthermore, in Trial B, when administered corticosteroids, azathioprine, vinca alkaloids or androgens before the screening visit, patients had to receive a stable IVIG dose and schedule for at least 3 weeks, 3 months, 8 weeks and 8 weeks, respectively, prior to screening visit.

Patients were excluded if they were pregnant or nursing females, had immune thrombocytopenia associated to other pathologies (secondary ITP) or had previous history or clinical findings of other autoimmune diseases.

\section{Study product}

Flebogamma 10\% DIF is manufactured from plasma from healthy donors. Purification procedure yields a high-purity unmodified $\mathrm{IgG}$ molecule with very little or undetectable content of accompanying proteins that might cause unexpected safety concerns [15]. Safety measures include testing at the level of individual plasma donation and plasma pools for nonreactivity for specific agents such as HIV-1 and HIV-2, hepatitis A virus, hepatitis $B$ virus and hepatitis $C$ virus, respectively, and $B 19$ virus using serological and nucleic acid amplification techniques. In addition, the manufacturing process of Flebogamma 10\% DIF includes pathogen elimination steps such as pasteurization, solvent-detergent treatment and nanofiltration $[16,17]$. The finished Flebogamma $10 \%$ DIF product is an immune globulin containing 5 or $10 \mathrm{~g}$ human normal immunoglobulin with a broad spectrum of antibodies against several infectious agents [18], stabilized with 2.5 or $5 \mathrm{~g} \mathrm{D}$-sorbitol in 50 or $100 \mathrm{ml}$ of water for injection, respectively.

\section{IVIG administration \& dosage}

IVIG dose in both trials was $1 \mathrm{~g} / \mathrm{kg}$ body weight per day over two consecutive days, for a total dose of $2 \mathrm{~g} / \mathrm{kg}$ body weight. In Trial $\mathrm{A}$, dose could also be administered at $400 \mathrm{mg} / \mathrm{kg}$ body weight per day over a period of five consecutive days, depending on the treatment the patient had received previously and to which he or she had responded. 
All infusions were intravenous, initiated at a rate of $0.01 \mathrm{ml} / \mathrm{kg}$ per minute for the first $30 \mathrm{~min}$ by means of an infusion pump. If tolerated (no AE observed which was associated to the infusion), the rate increased to $0.02 \mathrm{ml} / \mathrm{kg}$ per minute for the second $30 \mathrm{~min}$. Again, if tolerated, the rate was to be advanced to $0.04 \mathrm{ml} / \mathrm{kg}$ per minute for the third $30 \mathrm{~min}$. If the patient tolerated the infusion well, additional increments of $0.02 \mathrm{ml} / \mathrm{kg}$ per minute were made at 30 -min intervals up to a maximum of $0.10 \mathrm{ml} / \mathrm{kg}$ per minute.

\section{Efficacy assessments}

The rise in platelet count to $\geq 50 \times 10^{9} / 1$ (response), as a reliable predictor of clinical benefit, was considered the primary efficacy end point. The response rate was defined as the proportion of patients reaching the platelet count $\geq 50 \times 10^{\%} / 1$ during the study period (by day 90; Trial A) or by day 8 after starting IVIG treatment (Trial B). Secondary end points of efficacy included time to response, duration of response and bleeding signs.

Platelet counts were monitored after IVIG infusion and daily through days 6,10 and 14 (Trial A) or through day 8 (Trial $\mathrm{B})$, or until counts reached or exceeded $50 \times 10^{9} / 1$, whichever occurred first. Then, platelet counts were monitored every week/ month through final visit (day 90; Trial A) or every 3-5 days through final visit (day 30; Trial B). If a subject received alternative treatments with corticoids or immunosuppressive agents within 3 months of follow-up (Trial A), the platelet counts measured while these treatments were not taken into account and therefore not included in the study.

Regression of hemorrhage/bleeding episodes was determined, regardless of improvement in platelet counts, using a categorized rating scale, by the proportion of treated subjects who experienced improvement of clinical manifestation of hemorrhage/bleeding during the first 10 or 14 days (Trial A), or by the proportion of treated subjects with hemorrhage/bleeding on day 1 who improved their diathesis during the clinical follow-up period ending on day 15 (Trial B).

\section{Safety assessments}

Safety assessments included nature, severity and frequency of AEs during and after infusions; changes in vital signs (blood pressure, heart rate, body temperature and respiratory frequency) and clinically relevant changes in laboratory parameters (hematology, renal and hepatic functions) after the infusions; and patients' virology status (hepatitis A virus, hepatitis B virus, hepatitis $\mathrm{C}$ virus and HIV) at day 1 . Complete clinical follow-up was performed during the follow-up period.
All AEs occurring since the recruitment or first infusion visit and ended with the last follow-up visit for each subject were summarized by presenting infusion/subject incidences and percentages (each patient contributes a single count to a category) for each system organ class after medical coding according to the version 15.0 of MedDRA.

\section{Statistics}

For Trial A, the number of patients planned was not based on statistical conditions but rather on the recommendations of the EMA on evaluating IVIG preparations in ITP patients [19], which consider a study sufficient only when at least 15 subjects are enrolled. Thus, the goal was to recruit a total of 20 patients to compensate for possible withdrawals.

For Trial B, the sample size calculation was based on the primary efficacy end point (response rate) to detect $50 \%$ or fewer of treated patients that increase platelet count from $\leq 20 \times 10^{9} / 1$ (at day 1 ) to $\geq 50 \times 10^{9} / 1$ by day 8 , with a 0.05 one-sided significance level and $80 \%$ power. Thus, the goal was to recruit 75 patients to achieve at least 67 patients. The nQuery Advisor v5 (Statistical Solutions Ltd, Cork, Ireland) software was used for calculations.

All subjects who received at least one infusion of IVIG were included in the (modified) intent-to-treat (mITT) analysis population, used for efficacy and safety analysis and summaries. A per protocol (PP) population, consisting of the mITT population but excluding subjects with major protocol violations, was defined, and the primary analysis was repeated using the PP population. Summary statistics were provided for at least two groups: adults and children $(<18$-year old).

Demographic and baseline characteristics were summarized by using descriptive statistics. Absolute and relative frequencies of subjects in several categories were presented. For quantitative variables, mean, median and standard deviation were calculated. The 95\% CIs for the mean were computed using the t-distribution. In case of marked non-normal distribution, normalizing transformations might be applied. For qualitative variables, the proportions and $95 \%$ confidence limits were computed by the Clopper-Pearson method.

\section{Results \\ Patients \& IVIG administration}

A total of 76 patients who met the inclusion criteria were enrolled in the global assessment (mITT population: 18 in Trial A and 58 in Trial B), performed from 19 May 2008 (first patient enrolled) to 6 March 2012 (last patient completed). 
Seven subjects discontinued the study early (all in Trial B), five of them due to disease progression, one due to withdrawal of informed consent and one due to other reasons. At the end of the study, 69 patients were evaluated (18 in Trial A and 51 in Trial B).

Fourteen subjects were excluded (four in Trial A and ten in Trial B) because of protocol violation (PP population: 14 in Trial A and 48 in Trial B). Figure 1 shows the flow of patients through the overall analysis.

Across the studies 64 adults and 12 children were enrolled. All children came from Trial B. The patients' age range was from 3 to 77 years. Patients' distribution by sex was globally balanced $(51.3 \%$ females and $48.7 \%$ males). Just over half of the patients were Caucasian $(51.3 \%)$. Subject demographics by Trial are shown in Table 1.

All 76 subjects enrolled received at least one infusion (at any dose) of IVIG. Of the 18 subjects enrolled in Trial A, $11(61.1 \%)$ received the 2-day IVIG dosing regimen and $7(38.9 \%)$ received the 5-day regimen. All 18 patients (100\%) successfully completed the infusions and no subject terminated the study early. The total number of IVIG infusions administered in Trial A was 57. Four patients (three adults, one child) out of the 58 enrolled in Trial B received less than $80 \%$ of the planned dose, which was a protocol violation and were therefore excluded from the PP population. The total number of IVIG infusions administered in Trial B was 115.

\section{Analysis of efficacy}

The response rate (\% of patients reaching the platelet count $\geq 50 \times 10^{9} / 1$ by the established day) was $72.2 \%$ (95\% CI: $50.2-88.4 \%$ ) and $76.1 \%$ (95\% CI: 63.5-86.0\%) in Trials A and B, respectively, for adult mITT population. Values were somewhat lower and somewhat higher, respectively, in the PP population. Details are shown in Table 2. Percentage of responders

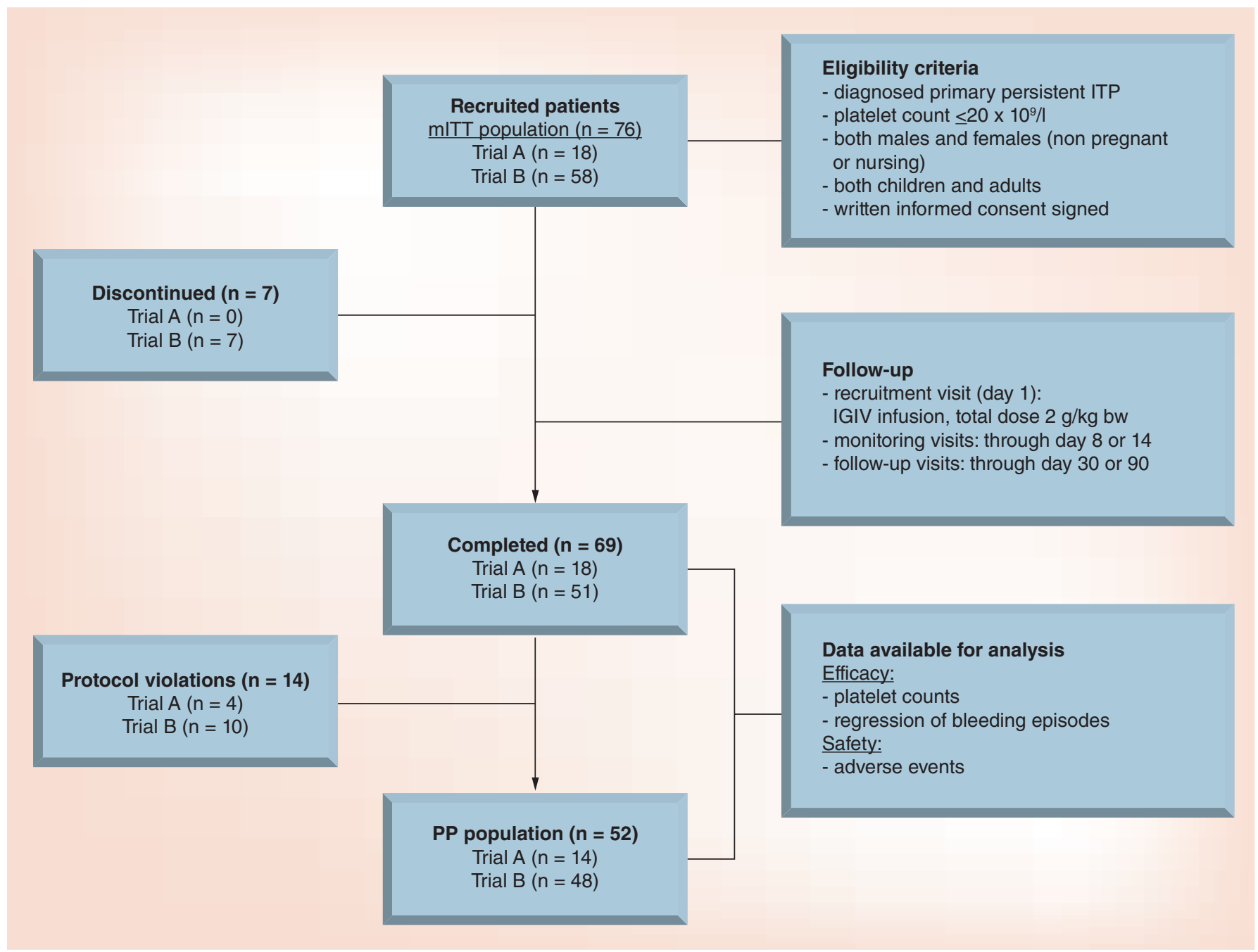

Figure 1. Flow of patients through the study.

ITP: Immune thrombocytopenic purpura; IVIG: Intravenous immune globulin; mITT: Modified intent-to-treat. 
Table 1. Demographic parameters.

\begin{tabular}{|c|c|c|c|}
\hline & \multirow{2}{*}{$\begin{array}{r}\text { Trial A } \\
\text { Adults }(n=18)\end{array}$} & \multicolumn{2}{|c|}{ Trial B } \\
\hline & & Adults $(n=46)$ & Children $(n=12)$ \\
\hline Age (years), mean $\pm S D$ & $43.7 \pm 19.1$ & $38.9 \pm 16.7$ & $11.5 \pm 3.9$ \\
\hline Male sex, n (\%) & $18(100)$ & $13(28.3)$ & $6(50.0)$ \\
\hline \multicolumn{4}{|l|}{ Geographic origin, n (\%) } \\
\hline Asian & 0 & $31(67.4)$ & 0 \\
\hline Caucasian & $18(100)$ & $12(26.1)$ & $9(75.0)$ \\
\hline South-American & 0 & $2(4.3)$ & $2(16.7)$ \\
\hline African-American & 0 & $1(2.2)$ & $1(8.3)$ \\
\hline
\end{tabular}

in children was $100 \%$ in all cases (see Table 2). For all subjects in Trial B (adult + children), the response rate was $81.0 \%$ (95\% CI: 70.6-89.0\%).

Results of secondary efficacy analysis are summarized in Table 3. Time to response in adult patients was around 2 days in both trials, with duration of around 10 days. Maximum platelet count was around $200 \times$ $10^{\%} / 1$. In children, time to response was shorter (around 1 day and half), duration was longer (around 2 weeks) and maximum platelet count was higher (around 335 $\left.\times 10^{9} / \mathrm{l}\right)$. The Kaplan-Meier plot of time-to-platelet count recovery is shown in Figure 2.
Among the 18 subjects in the mITT population of Trial A, 15 (83.3\%) experienced regression of clinical manifestation of hemorrhage/bleeding in the first 10 days of observation, and the number remained unchanged for the first 14 days of observation. In Trial B, 27 adults and 12 children showed hemorrhage/bleeding on day 1 . Of these, $24(88.9 \%)$ and $12(100 \%)$ improved their diathesis during the clinical follow-up period ending on day 15 . These results are summarized in Table 4. The Kaplan-Meier plot for time to regression of hemorrhage/bleeding is illustrated Figure 3.

Table 2 . Response rates ( $\%$ of patients reaching the platelet count $\geq 50 \times 10^{9} /$ by day 90 [Trial A] or by day 8 after starting intravenous immune globulin treatment [Trial B]).

\begin{tabular}{|c|c|c|c|}
\hline & Trial A & \multicolumn{2}{|c|}{ Trial B } \\
\hline & Adults & Adults & Children \\
\hline mITT population, $\mathrm{N}$ & 18 & 46 & 12 \\
\hline Responders, n (\%) & $13(72.2)$ & $35(76.1)$ & $12(100)$ \\
\hline $95 \% \mathrm{Cl}$ & $50.2-88.4$ & $63.5-86.0$ & $77.9-100$ \\
\hline PP population, $\mathrm{N}$ & 14 & 37 & 11 \\
\hline Responders, n (\%) & $9(64.3)$ & $33(89.2)$ & $11(100)$ \\
\hline $95 \% \mathrm{Cl}$ & $39.0-84.7$ & $76.9-96.2$ & $76.2-100$ \\
\hline
\end{tabular}

Table 3. Time to response and duration, maximum platelet count reached during response and time to be reached, in treatment responders (mean $\pm S D$ ).

\begin{tabular}{|c|c|c|c|}
\hline & \multirow{2}{*}{$\begin{array}{c}\text { Trial A } \\
\text { Adults }(n=13)\end{array}$} & \multicolumn{2}{|c|}{ Trial B } \\
\hline & & Adults $(n=35)$ & Children $(n=12)$ \\
\hline Time to response (days) $^{\dagger}$ & $\leq 2.1 \pm 1.3$ & $\leq 1.8 \pm 1.0$ & $\leq 1.4 \pm 0.5$ \\
\hline Duration of response (days) ${ }^{\ddagger}$ & $\geq 13.5 \pm 14.6$ & $\geq 9.6 \pm 7.8$ & $\geq 14.3 \pm 8.0$ \\
\hline Max. platelet count $\left(\times 10^{9} / \mathrm{I}\right)$ & $204.5 \pm 183.3$ & $205.3 \pm 146.1$ & $335.2 \pm 198.4$ \\
\hline Time to max. plat. count (days) & $\mathrm{n} / \mathrm{a}$ & $4.7 \pm 1.9$ & $5.3 \pm 1.2$ \\
\hline \multicolumn{4}{|c|}{$\begin{array}{l}{ }^{\dagger} \text { As the time to response was only assessed at the study visits, it is likely that a response occurred already before the visit and therefore the } \\
\text { symbol } \leq \text { is used. } \\
\text { 'The duration of response was also determined at the visit and not between two visits, so it is also likely that the response lasted beyond the } \\
\text { visit. Therefore, the symbol } \geq \text { is used. }\end{array}$} \\
\hline
\end{tabular}




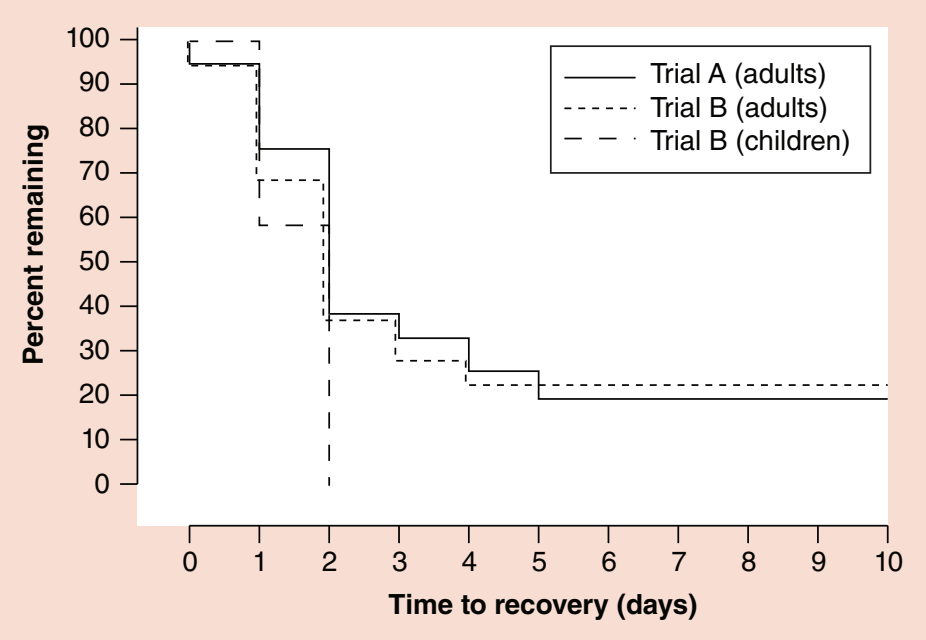

Figure 2. The Kaplan-Meier plot of time-to-platelet count recovery in all subjects (mITT population; Trial A: $n=18$ adults; Trial $B: n=46$ adults, $n=$ 12 children).

mITT: Modified intent-to-treat.

\section{Safety assessments}

An overall summary of the number and percentage of subjects with at least one AE (occurred after the start of IVIG infusion) and AEs by seriousness, severity and relatedness is presented in Table 5. Clinically significant abnormal laboratory test values, vital signs and physical findings were reported as AEs.

A total of 85 AEs were reported in 16 subjects $(88.9 \%)$ in the Trial A, and 257 AEs in 53 subjects (91.4\%) in the Trial B (137 AEs in 41 adult subjects [89.1\%] and 120 AEs in 12 children [100\%]). The most common AEs in Trial A were petechiae (12 subjects [66.7\%]), ecchymosis (eight subjects [44.4\%]), headache (seven subjects [38.9\%]) and pyrexia (four subjects [22.2\%]). In Trial $\mathrm{B}$, the most frequent $\mathrm{AE}$ was headache (38 subjects [65.5\%]), nausea (14 subjects [24.1\%]), pyrexia (12 subjects [20.7\%]) and chills (11 subjects [19.0\%]). Several AEs were much more frequent in children than adult subjects: nausea (66.7 vs $13.0 \%$ ), vomiting ( 41.7 vs $8.7 \%$ ) and hypotension (41.7 vs 2.2\%). A summary of the most common AEs potentially related to the product is shown in Table 6 .
Globally, most AEs were nonsevere (60 out of 70; $86 \%$; Table 5). Two children (16.7\%) and seven adult subjects experienced severe AEs (one in Trial A [5.6\%]) and six in Trial B [13.0\%]). AEs potentially related to IVIG treatment were reported by 38.9 and $41.4 \%$ (30.4\% of adults and $83.3 \%$ of children) of patients in Trials A and B, respectively.

Only five patients experienced serious AEs (Table 5), two adults in Trial A (three AEs) and three adults in Trial B (three AEs). The six serious AEs were: leukopenia and decreased hemoglobin in one subject, both of which were considered by the investigator as probably related to the study drug; thrombosis in one subject that was considered possibly related to the study drug; soft tissue inflammation in one subject, which was severe and deemed by the investigator as unrelated to study drug; and one event of headache in two subjects, which both were severe and considered possibly treatment related. All serious AEs were resolved without sequelae. No deaths have been reported in the clinical studies. No seroconversion in the viral markers was seen in any subject.

From a temporal perspective, of the total of 57 IVIG infusions in Trial A, the percent of them with AEs which occurred during the actual infusion was $10.5 \%$ (six infusions) and this percent increased to $24.6 \%$ (14 infusions) and $26.3 \%$ (15 infusions) for AEs that occurred up to 24- to 72-h postinfusion, respectively. In Trial B, 50.0\% of subjects (29/58) experienced at least one AE during infusion and this percent increased to $70.7 \%$ (41 subjects) and $72.4 \%$ (42 subjects) for AEs that occurred up to 24- to 72-h postinfusion, respectively.

\section{Discussion}

In recent years, the investigation and management of patients with ITP has varied widely and different consensus documents have been published to provide recommendations $[1,5,6]$. Overall, the treatment with IVIG is effective in elevating platelet count in approximately $65-85 \%$ of adult patients with ITP [9]. In this study, effectiveness and safety of Flebogamma $10 \%$

Table 4. Proportion of patients showing regression of hemorrhage/bleedings.

\begin{tabular}{|c|c|c|c|c|}
\hline \multirow{3}{*}{ Day of assessment } & \multicolumn{2}{|c|}{ Trial A } & \multirow{2}{*}{\multicolumn{2}{|c|}{\begin{tabular}{|l} 
Trial B \\
Day 15
\end{tabular}}} \\
\hline & \multirow[t]{2}{*}{ Day 10} & \multirow[t]{2}{*}{ Day 14} & & \\
\hline & & & Adults & Children \\
\hline $\mathrm{N}^{*}$ & 18 & 18 & 27 & 12 \\
\hline Patients improved, n (\%) & $15(83.3)$ & $15(83.3)$ & $24(88.9)$ & $12(100)$ \\
\hline $95 \% \mathrm{Cl}$ & $62.3-95.3$ & $62.3-95.3$ & $70.8-97.6$ & $73.5-100$ \\
\hline
\end{tabular}


DIF was found effective and safe in a broad worldwide patient population, through two similarly designed trials, for the treatment of primary ITP lasting at least 3-6 months, a condition considered as persistent ITP according to current terminology [1].

Both trials followed the IVIG dose recommended in children and adults with ITP with high risk of bleeding or prior to surgery to correct platelet count (2 g/ $\mathrm{kg}$ infused over 2-5 days) [2,6,9,20]. Although designs were not identical and geographic origin of populations was diverse, results obtained in both trials were highly comparable.

The rate of response (primary efficacy end point) in adult mITT patients was very similar in both Trials $A$ and B (72.2 and 76.1\%, respectively), in spite of the different definition of the end point. These rates were comparable to that reported for Flebogamma 5\% DIF (around 70\%) [14]. The pediatric responder rate was $100 \%$. The lower limit of the exact one-sided 95\% CI was 50.2 and 70.6\% (Trials A and B, respectively) which were larger than $50 \%$; and consequently, it can be accepted that greater than $50 \%$ of treated subjects increased platelet count from $\leq 20 \times 10^{9} / 1$ to $\geq 50 \times 10^{9} / 1$. Thus, the study results indicated that the primary efficacy end point of the study was met. It is reported in the literature that the response to IVIG in patients with ITP may vary with products, the dosing mode or the study design [2]. Therefore, any comparison should be made with caution. Overall, clinical efficacy results are consistent with published studies with other IVIG (ranging from 71 to $92 \%$ ) [21-29].

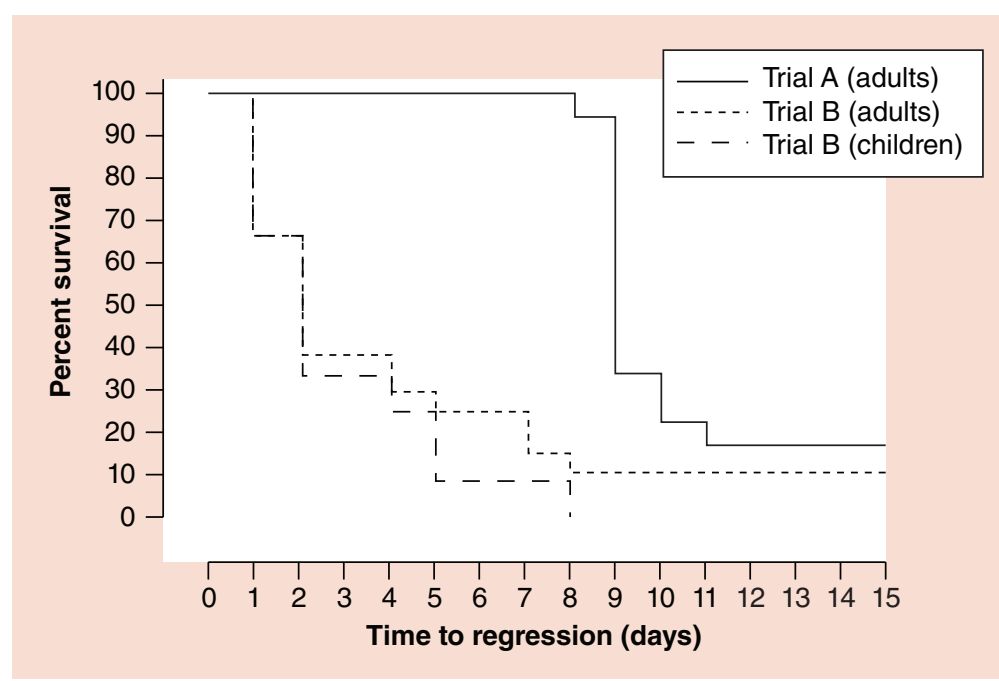

Figure 3. The Kaplan-Meier plot of time to hemorrhage/bleeding regression in all subjects (mITT population) in Trial $A(n=18)$ and in subjects that presented some sign of hemorrhage/bleeding at day 1 in Trial $B$ ( $n=27$ adults; $n=12$ children).

mITT: Modified intent-to-treat.

The secondary efficacy end point results provided further evidence for the efficacy of Flebogamma 10\% DIF in treating subjects with ITP. In both trials, time to response in adult patients was around 2 days, with duration of around 10 days. In general, response to IVIG was fast and transient as expected according to the literature $[2,3]$.

Furthermore, regarding clinical efficacy, a wide majority of patients who presented bleeding diathesis at screening (prior to first infusion), regardless

\section{Table 5. Subjects with at least one adverse event occurring between the start of intravenous immune globulin infusion visit and the last follow-up visit.}

\begin{tabular}{|c|c|c|c|}
\hline & \multirow{2}{*}{$\begin{array}{l}\text { Trial A } \\
\text { Adults }(n=18)\end{array}$} & \multicolumn{2}{|c|}{ Trial B } \\
\hline & & Adults $(n=46)$ & Children $(n=12)$ \\
\hline Subjects with at least one $A E, n(\%)$ & $16(88.9)$ & $41(89.1)$ & $12(100)$ \\
\hline \multicolumn{4}{|l|}{ Seriousness, n (\%) } \\
\hline Nonserious & $16(88.9)$ & $38(93.5)$ & $12(100)$ \\
\hline Serious & $2(11.1)$ & $3(6.5)$ & 0 \\
\hline \multicolumn{4}{|l|}{ Severity, $n(\%)$} \\
\hline Mild & $5(27.8)$ & $27(58.7)$ & $4(33.3)$ \\
\hline Moderate & $10(55.6)$ & $8(17.4)$ & $6(50.0)$ \\
\hline Severe & $1(5.6)$ & $6(13.0)$ & $2(16.7)$ \\
\hline \multicolumn{4}{|l|}{ Relatedness, n (\%) } \\
\hline Unrelated/doubtful & $9(50.0)$ & $5(10.9)$ & 0 \\
\hline Unlikely/conditional & 0 & $3(6.5)$ & 0 \\
\hline Possible & 0 & $19(41.3)$ & $2(16.7)$ \\
\hline Probable/definite & $7(38.9)$ & $14(30.4)$ & $10(83.3)$ \\
\hline AE: Adverse event. & & & \\
\hline
\end{tabular}




\begin{tabular}{|c|c|c|c|c|c|c|}
\hline \multirow{3}{*}{$\begin{array}{l}\text { System organ class } \\
\text { preferred term }\end{array}$} & \multirow{2}{*}{\multicolumn{2}{|c|}{$\begin{array}{c}\text { Trial A } \\
\text { Adults }(n=18)\end{array}$}} & \multicolumn{4}{|c|}{ Trial B } \\
\hline & & & \multicolumn{2}{|c|}{ Adults $(n=46)$} & \multicolumn{2}{|c|}{ Children $(n=12)$} \\
\hline & Subjects, n (\%) & AEs, $n$ & Subjects, n (\%) & AEs, $n$ & Subjects, n (\%) & AEs, $n$ \\
\hline \multicolumn{7}{|l|}{ Nervous system disorders } \\
\hline Headache & $5(27.8)$ & 12 & $24(52.2)$ & 34 & $11(91.7)$ & 19 \\
\hline Dizziness & 0 & 0 & $4(8.7)$ & 4 & 0 & 0 \\
\hline Radiculopathy & $1(5.6)$ & 1 & 0 & 0 & 0 & 0 \\
\hline \multicolumn{7}{|c|}{ General disorders and administration site conditions } \\
\hline Pyrexia & $3(16.7)$ & 3 & $8(17.4)$ & 11 & $4(33.5)$ & 5 \\
\hline Chills & $2(11.1)$ & 2 & $7(15.2)$ & 8 & $4(33.5)$ & 4 \\
\hline Fatigue & $1(5.6)$ & 2 & 0 & 0 & 0 & 0 \\
\hline \multicolumn{7}{|l|}{ Gastrointestinal disorders } \\
\hline Nausea & $1(5.6)$ & 1 & $4(8.7)$ & 4 & $8(66.7)$ & 9 \\
\hline Vomiting & $1(5.6)$ & 1 & $4(8.7)$ & 4 & $5(41.7)$ & 5 \\
\hline Diarrhea & 0 & 0 & $3(6.5)$ & 3 & 0 & 0 \\
\hline Vascular disorders & $2(11.1)$ & 4 & $4(8.7)$ & 4 & $3(25)$ & 5 \\
\hline $\begin{array}{l}\text { Skin and subcutaneous } \\
\text { tissue disorders }\end{array}$ & $2(11.1)$ & 3 & $3(6.5)$ & 3 & $3(25)$ & 3 \\
\hline $\begin{array}{l}\text { Musculoskeletal and } \\
\text { connective tissue } \\
\text { disorders }\end{array}$ & $1(5.6)$ & 1 & $2(4.3)$ & 2 & $2(16.7)$ & 2 \\
\hline Other disorders & $2(11.1)$ & 3 & $1(2.2)$ & 2 & $2(16.7)$ & 2 \\
\hline $\begin{array}{l}\text { Abnormal vital signs/ } \\
\text { laboratory parameters }\end{array}$ & $1(5.6)$ & 1 & $7(15.2)$ & 9 & $3(25)$ & 13 \\
\hline AE: Adverse event. & & & & & & \\
\hline
\end{tabular}

of whether they were responders in terms of platelet counts, experienced improvement in diathesis in 14-15 days of assessment $(>80 \%$ of the adults in both Trials A and $\mathrm{B}$, and $100 \%$ of children). These results are consistent with the data described for Flebogamma 5\% DIF (90\% of patients with improvement) [14].

In general, incidence of AEs reported in clinical trials of IVIG products varies widely due to different characteristics of the trials such as IgG contents of the products, premedication, maximal infusion rate and patient population being studied. Generally, AEs such as headache, nausea, vomiting, pyrexia, chills or back pain are common and expected after treatment with IVIG. Serious, but rare, AEs have also been reported, including acute renal failure, severe immediate hypersensitivity reactions and thromboembolic complications [30-32].

In this study, $90.8 \%$ of all subjects ( $87.7 \%$ in adults) reported at least one AE between the start of study drug infusion and the final visit, a rate only slightly higher than that observed with Flebogamma 5\% DIF
(80.0\%) [14]. Although the percentage of subjects reporting AEs varies widely (from 43 to $85 \%$ ) among studies with $5 \%[22-24,27,29]$ and $10 \%[21,25,26,28,33]$ IVIG products, it is known that rates of AEs are higher in more concentrated IVIG products [31,32]. The most common AEs were headache, pyrexia, nausea, chills, contusion, hypotension and vomiting, which are consistent with the AEs typically observed with IVIG infusions [32]. Regarding the observed differences in patterns of AEs between children and adult subjects, it should be pointed out that the number of children was much smaller than that of adult subjects.

The majority of subjects with at least one AE reported them with a maximum severity of mild or moderate. From a temporal perspective, most of the events within the first $72 \mathrm{~h}$ after study drug infusion were clustered within the first $24-\mathrm{h}$ poststudy drug infusion. These results showed that there was an adequate tolerance for Flebogamma 10\% DIF during and after infusions.

Of the treatment-related AEs reported, the most common were headache, followed by pyrexia, nausea, 
chills and vomiting. These AEs that were deemed potentially related to study drug and were comparable to those observed with Flebogamma 5\% DIF [14]. There were no deaths and no subjects who discontinued the study early due to an AE. The serious AEs reported were all in adult subjects, which resolved without sequele. The nature and severity of these AEs are consistent with what are expected for IVIG therapies [32].

\section{Conclusion}

The results from this analysis indicate that IVIG Flebogamma $10 \%$ DIF, administered for a total dose of $2 \mathrm{~g} / \mathrm{kg}$, was effective and safe in treating subjects with primary ITP.

\section{Future perspective}

Although thrombopoietic agents and immunomodulators/immunosupresors are therapies available for ITP, it is unlikely that they may shift IVIG as an alternative in the setting of ITP for its target patients: those adults and children with high risk of bleeding and those needing to quickly raise platelet count to hemostatic levels due to a surgery procedure. In this context, a large prospective trial comparing steroids or rituximab with IVIG will is not expected in the short- to mid-term. In light of the eventual production of new IVIG, clinical investigation will go on to confirm their efficacy and safety for the mentioned therapeutic indication.

\section{Acknowledgements}

The following investigators of the Flebogamma ${ }^{\circledR}$ DIF in ITP Study Group are acknowledged: MT Álvarez (Hospital Universitario La Paz, Madrid, Spain); C-L Fu (Cleveland Clinic Florida, Weston, FL, USA); MJ John (Christian Medical College, Ludhiana, Punjab, India); A Julià (Hospital Universitari Vall d'Hebron, Barcelona, Spain); R Kaczmarski (The Hillingdon Hospital, Middlesex, UK); G Kato (Scottsdale Medical Specialists Ltd., Scottsdale, AZ, USA); N Parikh (Connecticut Children's Medical Center, Hartford, CT, USA); E Pustovaya (Research Centre of Russian Academy of Medical Science, Moscow, Russia); JA Rodríguez García (Hospital de León, León, Spain); C Ross (St. John's Medical College Hospital, Bangalore, Karnataka, India); V Sandoval (Hospital de León,
León, Spain); MÁ Sanz (Hospital Universitario La Fe, Valencia, Spain); C Shah (Shalby Hospitals, Ahmedabad, Gujarat, India); M Torres (Cook Children's Medical Center, Fort Worth, TX, USA). The following centers are acknowledged for patient recruitment: Children's Hospital of Orange County, Orange, CA, USA; Kodlikeri Memorial Hospital, Aurangabad, Maharashtra, India; Lombardi Cancer Center, Washington, DC, USA; Netaji Subhash Chandra Bose Cancer Research Institute, Kolkata, West Bengal, India; CTO Breslin Cancer Center, Lansing, MI, USA; Children's Hospital of Oklahoma, Oklahoma City, OK, USA; Mount Sinai Hospital, New York City, NY, USA; University of Medicine and Dentistry of New Jersey-Robert Wood Johnson Medical School, New Brunswick, NJ, USA; Kenmar Research Institute LLC, Los Angeles, CA, USA; Private Hematology/Oncology Practice, Lake Worth, FL, USA; Children's Hospital-Louisiana State University School of Medicine, New Orleans, LA, USA; Kalamazoo Hematology and Oncology, Kalamazoo, MI, USA and St. Joseph's Children's Hospital of Tampa, Tampa, FL, USA.

\section{Financial \& competing interests disclosure}

This study was funded by Grifols, manufacturer of Flebogam$m a^{\circledR} 10 \%$ DIF and Flebogamma 5\% DIF. J Navarro-Puerto is an employee of Grifols. The authors have no other relevant affiliations or financial involvement with any organization or entity with a financial interest in or financial conflict with the subject matter or materials discussed in the manuscript apart from those disclosed. Writing assistance was utilized in the production of this manuscript. J Bozzo (Grifols) is acknowledged for medical writing and editorial support in the preparation of the manuscript. Content Ed Net, Madrid (Spain) is acknowledged for editorial assistance.

\section{Ethical conduct of research}

The trials were designed and monitored in compliance with the ethical principles of the World Medical Assembly (Helsinki, 1964) and subsequent revisions, and the applicable guidance documents set forth by regulatory bodies [16] for the clinical investigation of IVIG products in ITP. Written informed consent from each subject participating in the study was obtained. National and local independent ethics committee approved both the protocol and the associated informed consent and information sheets.

\section{Executive summary}

- Intravenous immune globulin (IVIG) treatment at $2 \mathrm{~g} / \mathrm{kg}$ infused over 2-5 days has been previously shown to be efficacious for children and adults with immune thrombocytopenic purpura (ITP).

- ITP subjects were enrolled in two similarly designed open-label, non-controlled, multicenter clinical trials. IVIG (Flebogamma ${ }^{\circledR} 10 \%$ DIF) was infused to enrolled patients for a total dose of $2 \mathrm{~g} / \mathrm{kg}$ (over 2 or 5 days).

- In a follow-up of 1-3 months there was evidence of improved response rate (reaching platelet count $\geq 50 \times$ $10 \% / 1)$ and improved in bleeding diathesis.

- Potential treatment-related adverse events were similar to those observed with other $10 \%$ IVIG products.

- The few serious adverse events resolved without sequelae.

- Flebogamma $10 \%$ DIF, was effective and safe in treating subjects with ITP. 


\section{Open access}

This work is licensed under the Attribution-NonCommercialNoDerivatives 4.0 Unported License. To view a copy of this license, visit http://creativecommons.org/licenses/by-nc-nd/4.0

\section{References}

Papers of special note have been highlighted as:

- of interest; $\bullet$ of considerable interest

1 Rodeghiero F, Stasi R, Gernsheimer T et al. Standardization of terminology, definitions and outcome criteria in immune thrombocytopenic purpura of adults and children: report from an international working group. Blood 113(11), 2386-2393 (2009).

2 British Committee for Standards in Haematology General Haematology Task Force. Guidelines for the investigation and management of idiopathic thrombocytopenic purpura in adults, children and in pregnancy. Br. J. Haematol. 120(4), 574-596 (2003).

-. Guideline that provides a rational approach to the laboratory investigation and management of patients with immune thrombocytopenic purpura (ITP), including pregnant and nonpregnant adults and children, and patients with refractory disease.

3 Chong BH, Ho SJ. Autoimmune thrombocytopenia. J. Thromb. Haemost. 3(8), 1763-1772 (2005).

4 Cines DB, Blanchette VS. Immune thrombocytopenic purpura. N. Engl. J. Med. 346(13), 995-1008 (2002).

5 Neunert C, Lim W, Crowther M, Cohen A, Solberg L Jr, Crowther MA. The American Society of Hematology 2011 evidence-based practice guideline for immune thrombocytopenia. Blood 117(16), 4190-4207 (2011).

6 Provan D, Newland A. Fifty years of idiopathic thrombocytopenic purpura (ITP): management of refractory ITP in adults. Br. J. Haematol. 118(4), 933-944 (2002).

- A review that defines the ITP disorder, discusses the underlying immunological features, summarizes the standard therapy and reviews therapies under development.

7 Vesely SK, Perdue JJ, Rizvi MA, Terrell DR, George JN. Management of adult patients with persistent idiopathic thrombocytopenic purpura following splenectomy: a systematic review. Ann. Intern. Med. 140(2), 112-120 (2004).

8 George JN, el Harake MA, Aster RH. Thrombocytopenia due to enhanced platelet destruction by immunologic mechanism. In: Williams Hematology. Beutler E, Lichtmann MA, Boller BA, Kipps TJ (Eds). McGraw Hill, New York, 1315-1355 (1995).

9 Stasi R, Provan D. Management of immune thrombocytopenic purpura in adults. Mayo Clin. Proc. 79(4), 504-522 (2004).

10 Pavone P, Passaniti E, Taibi R et al. Intravenous immunoglobulin therapy when plasmapheresis fails in thrombotic thrombocytopenic purpura associated with severe ADAMTS 13 deficiency in childhood: a case report. Eur. J. Inflamm. 11(1), 291-296 (2013).

11 Kazatchkine MD, Kaveri SV. Immunomodulation of autoimmune and inflammatory diseases with intravenous immune globulin. N. Engl. J Med. 345(10), 747-755 (2001).

-• Describes the mechanisms of action and effectiveness of intravenous immune globulin in the treatment of autoimmune disorders such as ITP.

12 Zuercher AW, Spirig R, Baz MA, Kasermann F. IVIG in autoimmune disease - potential next generation biologics. Autoimmun. Rev. 15(8), 781-785 (2016).

13 Cherin P, Belizna C, Cartry O et al. Long-term subcutaneous immunoglobulin use in inflammatory myopathies: a retrospective review of 19 cases. Autoimmun. Rev 15(3), 281-286 (2016).

14 Julia A, Kovaleva L, Loria S et al. Clinical efficacy and safety of Flebogamma ${ }^{\circledR}$ DIF, a new high-purity human intravenous immunoglobulin, in adult patients with chronic idiopathic thrombocytopenic purpura. Transfus. Med. 19(5), 260-268 (2009).

- A previous study that evidenced efficacy of Flebogamma ${ }^{\circledR}$ DIF ( $5 \%$ in that case) in the treatment of ITP.

15 José M, Marzo N, Bono M et al. Pasteurization inactivates clotting enzymes during Flebogamma ${ }^{\circledR}$ and Flebogamma ${ }^{\circledR}$ DIF production. WebmedCentral Immunotherapy 2(5), 001917 (2011).

16 Caballero S, Nieto S, Gajardo R, Jorquera JI. Viral safety characteristics of Flebogamma ${ }^{\circledR}$ DIF, a new pasteurized, solvent-detergent treated and Planova $20 \mathrm{~nm}$ nanofiltered intravenous immunoglobulin. Biologicals 38(4), 486-493 (2010).

- Study that points out the high viral safety profile achieved with the pasteurization, solvent/detergent treatment and planova nanofiltration in the manufacturing process of Flebogamma DIF.

17 Diez JM, Caballero S, Belda F, Otegui M, Gajardo R, Jorquera JI. Capacity of the manufacturing process of Flebogamma ${ }^{\circledR}$ DIF, a new human high purity intravenous immunoglobulin, to remove a TSE model-agent. Biologicals 38(6), 670-674 (2010).

18 Marzo N, Herrerias A, López M, Jorquera JI. Broad spectra of antibodies against different pathogens in Flebogamma ${ }^{\circledR}$ DIF. Proceedings of the 15th Meeting of the European Society Immunodeficiencies - ESID 2012; Florence, Italy. October 3-6, 2012. 1-4.

19 European Medicines Agency (EMA), Committee for Medicinal Products for Human Use (CHMP). Guideline on the clinical investigation of human normal immunoglobulin for intravenous administration (IVIG). (2010). www.ema. europa.eu/docs/en_GB/document_library/Scientific_ guideline/2009/10/WC500004766.pdf.

20 Bierling P, Godeau B. Intravenous immunoglobulin and autoimmune thrombocytopenic purpura: 22 years on. Vox. Sang. 86(1), 8-14 (2004).

21 Bussel JB, Eldor A, Kelton JG et al. IGIV-C, a novel intravenous immunoglobulin: evaluation of safety, efficacy, mechanisms of action, and impact on quality of life. Thromb. Haemost. 91(4), 771-778 (2004).

22 Colovic M, Dimitrijevic M, Sonnenburg C, Suvajdzic N, Donfrid M, Bogdanovic A. Clinical efficacy and safety of 
a novel intravenous immunoglobulin preparation in adult chronic ITP. Hematol. J. 4(5), 358-362 (2003).

Dash CH, Gillanders KR, Stratford Bobbitt ME, Gascoigne EW, Leach SJ. Safety and efficacy of Gammaplex ${ }^{\circledR}$ in idiopathic thrombocytopenic purpura (ClinicalTrials.gov - NCT00504075). PLoS ONE 9(6), e96600 (2014).

24 Newland AC, Burton I, Cavenagh JD et al. Vigam-S, a solvent/detergent-treated intravenous immunoglobulin, in idiopathic thrombocytopenic purpura. Transfus. Med. 11(1), 37-44 (2001).

25 Robak T, Salama A, Kovaleva L et al. Efficacy and safety of Privigen, a novel liquid intravenous immunoglobulin formulation, in adolescent and adult patients with chronic immune thrombocytopenic purpura. Hematology 14(4), 227-236 (2009).

26 Robak T, Mainau C, Pyringer B et al. Efficacy and safety of a new intravenous immunoglobulin $10 \%$ formulation (octagam ${ }^{\circledR} 10 \%$ ) in patients with immune thrombocytopenia. Hematology 15(5), 351-359 (2010).

van der Meer JW, van Beem RT, Robak T, Deptala A, Strengers PF. Efficacy and safety of a nanofiltered liquid intravenous immunoglobulin product in patients with primary immunodeficiency and idiopathic thrombocytopenic purpura. Vox. Sang. 101(2), 138-146 (2011).
28 Varga G, Volkova Z, Leibl H et al. Efficacy and safety of the new intravenous immunoglobulin IGIV $10 \%$ in adults with chronic idiopathic thrombocytopenic purpura. Transfus. Med. Hemother. 33(6), 509-514 (2006).

29 Wolf HH, Davies SV, Borte M et al. Efficacy, tolerability, safety and pharmacokinetics of a nanofiltered intravenous immunoglobulin: studies in patients with immune thrombocytopenic purpura and primary immunodeficiencies. Vox. Sang. 84(1), 45-53 (2003).

30 Katz U, Shoenfeld Y. Review: intravenous immunoglobulin therapy and thromboembolic complications. Lupus 14(10), 802-808 (2005).

31 Orbach H, Katz U, Sherer Y, Shoenfeld Y. Intravenous immunoglobulin: adverse effects and safe administration. Clin. Rev. Allergy Immunol. 29(3), 173-184 (2005).

32 Pierce LR, Jain N. Risks associated with the use of intravenous immunoglobulin. Transfus. Med. Rev. 17(4), 241-251 (2003).

- A report to assist prescribers in the identification, management and proposed strategies to minimize the risks of intravenous immune globulin.

33 Bussel JB, Hanna K. Safety and tolerability of a novel chromatography-based intravenous immunoglobulin when administered at a high infusion rate in patients with immune thrombocytopenic purpura. Am. J. Hematol. 82(3), 192-198 (2007). 
